\title{
Orbital Localization, Charge Transfer, and Band Gaps in Semilocal Density-Functional Theory
}

Rickard Armiento and Stephan Kümmel

\section{Linköping University Post Print}

\section{Tweet}

N.B.: When citing this work, cite the original article.

Original Publication:

Rickard Armiento and Stephan Kümmel, Orbital Localization, Charge Transfer, and Band Gaps in Semilocal Density-Functional Theory, 2013, Physical Review Letters, (111), 3, 036402-1-036402-5.

http://dx.doi.org/10.1103/PhysRevLett.111.036402

Copyright: American Physical Society http://www.aps.org/ 


\title{
Orbital Localization, Charge Transfer, and Band Gaps in Semilocal Density-Functional Theory
}

\author{
R. Armiento \\ Department of Physics, Chemistry and Biology (IFM), Linköping University, SE-58183 Linköping, Sweden
}

S. Kümmel

Theoretische Physik IV, Universität Bayreuth, D-95440 Bayreuth, Germany

(Received 12 December 2012; published 17 July 2013)

\begin{abstract}
We derive an exchange energy functional of generalized gradient form with a corresponding potential that changes discontinuously at integer particle numbers. The functional is semilocal, yet incorporates key features that are connected to the derivative discontinuity of Kohn-Sham density-functional theory. We validate our construction for several paradigm systems and explain how it addresses central well-known deficiencies of antecedent semilocal methods, i.e., the description of charge transfer, properly localized orbitals, and band gaps. We find, e.g., an improved shell structure for atoms, eigenvalues that more closely correspond to ionization energies, and an improved description of band structure where localized states are lowered in energy.
\end{abstract}

DOI: 10.1103/PhysRevLett.111.036402

PACS numbers: 71.15.Mb, 31.15.E-

In Kohn-Sham (KS) density-functional theory (DFT) $[1,2]$ the fundamental limitation on the accuracy of computationally predicted properties is set by the approximation used for the universal exchange-correlation (xc) functional $E_{\mathrm{xc}}[n]$ with $n(\mathbf{r})$ the electron density. Currently available approximations allow a high general accuracy and low computational expense for applications throughout physics, chemistry, and biology. Nevertheless, a reoccurring theme in the prior literature is a set of hallmark deficiencies: an inaccurate description of charge transfer and charge distribution in separated systems, too small band gaps, and over-delocalized orbitals. There is a broad multidisciplinary interest for understanding these deficiencies and addressing them.

These hallmark deficiencies are closely related to a set of important exchange features completely omitted in normal semilocal approximations, e.g., the derivative discontinuity (DD) [3] and the relative offset between potentials for well-separated subsystems (cf., e.g., Ref. [4]). Such features can be achieved with orbital functionals [5], but at a considerable computational cost. It has so far been unclear if it is at all possible to address these shortcomings within semilocal DFT. In the present work we construct a functional of generalized gradient approximation (GGA) [6] form that includes the missing exchange features. Thus, all of the listed hallmark deficiencies can be addressed in DFT at the computational expense of a usual GGA calculation.

Some prior works have modeled important exchange features directly in the potential, rather than via the functional derivative of an energy functional [7-13]. A recent model potential of Becke and Johnson (BJ) [14] has with various modifications shown improved polarizabilities [15], band gaps [16,17], and atomic and molecular properties $[18,19]$. It was discovered that one of its decisive positive aspects is that its asymptotic limiting value outside a finite system depends on the eigenvalue of the highest occupied orbital (HOMO), but not its occupation number $[14,15]$. A postcorrective shift that makes the potential asymptotically go to zero gives precisely the type of discontinuous constant shift at integer particle numbers that is associated with the DD [3,9,11,14,15]. However, no energy functional corresponding to the BJ potential exists $[13,20]$.

In the following we derive an expression for $E_{x}^{\mathrm{SL}}$ that is semilocal (SL) in the density and at the same time has a functional derivative $v_{x}^{\mathrm{SL}}$ that shows all of the attractive properties of the BJ potential. We start from the GGA form [6]

$$
E_{x}^{\mathrm{SL}}=A_{x} \int n(\mathbf{r})^{4 / 3} F(s) d^{3} \mathbf{r},
$$

where $F(s)$ is the exchange enhancement factor, $A_{x}=-(3 / 4)(3 / \pi)^{1 / 3}$ in Hartree atomic units, and $s=$ $|\nabla n(\mathbf{r})| /\left(2\left(3 \pi^{2}\right)^{1 / 3} n^{4 / 3}(\mathbf{r})\right)$. The asymptotic density far outside a finite system is [7]

$$
n(\mathbf{r}) \rightarrow m_{I} C e^{-2 \sqrt{-2 \epsilon_{I}^{0}} r},
$$

where $C$ is a system-dependent normalization constant, $m_{i}$ is the occupation number of orbital $i$, and $i=I$ is the index of the highest occupied orbital. To allow for the possibility that the asymptotic potential of Eq. (2) may be nonzero, we write $\epsilon_{I}^{0}=\epsilon_{I}-\lim _{|\mathbf{r}| \rightarrow \infty} v_{x}^{\mathrm{SL}}(\mathbf{r})$ with $\epsilon_{I}$ the KS HOMO eigenvalue. Using this density we get an expression for the GGA potential in the long-range asymptotic limit [Eq. (37) of Ref. [7]].

This long-range asymptotic limit is decisive for the further considerations: in order to achieve the same positive features as the $\mathrm{BJ}$ potential, we have to mimic the 
asymptotic properties of the $\mathrm{BJ}$ potential; i.e., $v_{x} \rightarrow$ $\sim \sqrt{-\epsilon_{I}^{0}}$ [15]. The first important step in our construction is to note that the product $n^{1 / 3} s$ achieves this limit: when Eq. (2) is inserted into $n^{1 / 3} s$ one finds $n^{1 / 3} s=$ $\sqrt{-2 \epsilon_{I}^{0}} /\left(3 \pi^{2}\right)^{1 / 3}$. The second important step is that we can now use this condition to derive a differential equation for $F(s)$ by equating the known GGA-form expression for the asymptotic potential to the desired form $-A_{x} b_{1} n^{1 / 3} s$. Here, a constant $b_{1}$ that will be determined later was introduced. Solving this differential equation to leading order in $s$ we find the solution

$$
F(s)=B_{1} s \ln (s) \text {, }
$$

with $B_{1}=3 b_{1}$. (The Supplemental Material [21] and Refs. [22-24] present all the steps in detail.) This is a main result of this Letter: a normal semilocal GGA-type energy functional achieves the desired asymptotic behavior for $\boldsymbol{v}_{x}^{\mathrm{SL}}$ if $F(s)$ diverges as $\sim s \ln (s)$.

To find an expression for $F(s)$ that is reasonable for all $s$, the term in Eq. (3) alone is too limited. It is not trivial to extend the above formalism to add asymptotic terms with system-independent prefactors (i.e., $-1 / r$ ). However, the leading term for the asymptotic behavior outside the surface of a half-infinite bulk system is $v_{x} \rightarrow-c / z$ with $c$ a system-dependent prefactor and $z$ the distance to the surface [25]. For the asymptotic density in this limit we find $\ln (s) \rightarrow$ const $+2 \sqrt{-2 \epsilon_{I}^{0}} z / 3$. Similar steps as above, which equate the asymptotic potential to $-A_{x} B_{2} n^{1 / 3} s / \ln (s)$ (i.e., to first order $\sim \sqrt{-\epsilon_{I}^{0}} / z$ ) give another differential equation for $F(s)$ with the solution

$$
F(s)=B_{2} s \ln (\ln (s)) \text {. }
$$

This term diverges more slowly than Eq. (3) in $s$ and can be used alongside that term. The resulting asymptote $F(s) \rightarrow$ $B_{1} s \ln (s)+B_{2} s \ln (\ln (s))$ can be turned into a well-behaved $F(s)$ for all $s$ with a slight modification that recovers the gradient expansion $F(s)=1+\mu_{\mathrm{GE}} s^{2}$ with $\mu_{\mathrm{GE}}=10 / 81$ for small $s[26,27]$

$$
F(s)=1+B_{1} s \ln (1+s)+B_{2} s \ln (1+\ln (1+s)),
$$

with $B_{2}=\mu_{\mathrm{GE}}-B_{1}$. Equation (7) further below reveals that the remaining constant $B_{1}$ sets the strength of the derivative discontinuity. Similar parameters are present in many schemes that address exchange deficiencies in functionals, e.g., the mixing parameter for hybrid functionals [28-32] and the $U$ parameter in DFT $+U$ [33-36].

Returning to the asymptote outside a finite system, an asymptotic expansion of $v_{x}^{\mathrm{SL}}$ has a constant leading term, a term $\sim \ln (r) / r$, a system-independent term $\sim 1 / r$, and further system-dependent terms of the same order. We can achieve that the system-independent term becomes exactly $-1 / r$ by choosing

$$
B_{1}=(3 / 5) \mu_{\mathrm{GE}}+8 \pi / 15 \text {. }
$$

This choice is further corroborated by the tests below, where it gives a reasonable size and behavior of the DD. The resulting $E_{x}^{\mathrm{SL}}$ fulfills uniform density scaling [37] and the second order gradient expansion [26,27], but not the local Lieb-Oxford bound (constraint ' $g$ ' of Ref. [38]).

In Fig. 1 we see how this $F(s)$ stays close to the functional by Perdew, Burke, and Ernzerhof (PBE) [38] for small $s$, but increases more quickly beyond $s \gtrsim 0.4$ to achieve the necessary asymptote proportional to $s \ln (s)$. The divergence of $F(s)$, as opposed to most other semilocal functionals with a $v_{x} \rightarrow 0$, is an important feature of our scheme.

We have thus finished the derivation of the functional and now review the beneficial properties that we have achieved by making the asymptotic potential depend on the HOMO eigenvalue. First, we derive the value that our potential asymptotically takes and find

$$
\lim _{|\mathbf{r}| \rightarrow \infty} v_{x}^{\mathrm{SL}}(\mathbf{r})=\left(A_{x}^{2} Q_{x}^{2} / 2\right)\left(1 \pm \sqrt{1-4 \epsilon_{I}^{\mathrm{SL}} /\left(A_{x}^{2} Q_{x}^{2}\right)}\right),
$$

where $Q_{x}=\left(\sqrt{2} /\left(3\left(3 \pi^{2}\right)^{1 / 3}\right)\right) B_{1}$, and the minus sign in front of the square root applies to the case $\epsilon_{I}^{\mathrm{SL}}<0$ (see the Supplemental Material [21] for an expanded discussion). With this knowledge we can construct a potential

$$
v_{x}^{0}(\mathbf{r})=v_{x}^{\mathrm{SL}}(\mathbf{r})+v_{x}^{\mathrm{DD}}, \quad v_{x}^{\mathrm{DD}}=-\lim _{|\mathbf{r}| \rightarrow \infty} v_{x}^{\mathrm{SL}}(\mathbf{r}),
$$

that asymptotically goes to zero and has eigenvalues $\left\{\epsilon_{i}^{0}\right\}=\left\{\epsilon_{i}^{\mathrm{SL}}+\boldsymbol{v}_{x}^{\mathrm{DD}}\right\}$. Note that for finding the selfconsistent Kohn-Sham orbitals it makes no difference whether one uses $v_{x}^{\mathrm{SL}}(\mathbf{r})$ or $\boldsymbol{v}_{x}^{0}(\mathbf{r})$. However, due to the dependence on $\epsilon_{I}^{\mathrm{SL}}$ the asymptotic limiting value of $v_{x}^{\mathrm{SL}}(\mathbf{r})$ can change discontinuously and the realigned potential $v_{x}^{0}$ then undergoes a uniform discontinuous shift. It thus reproduces the feature associated with the DD in the exact exchange (EXX) functional. The size of the DD $\Delta_{x}$ can be directly calculated as the difference in the two $v_{x}^{\mathrm{DD}}$

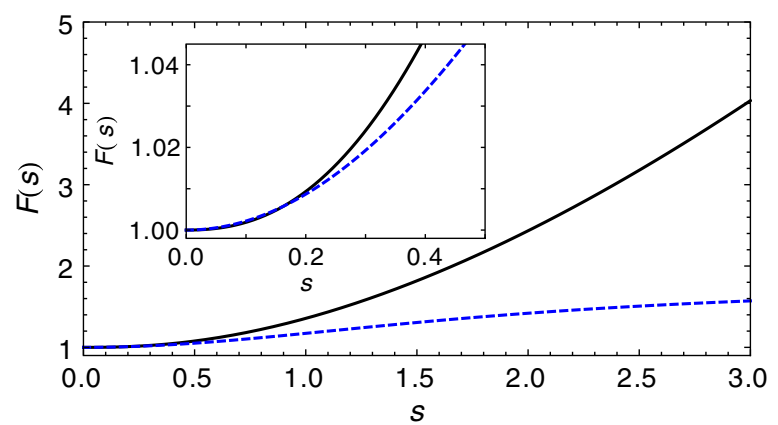

FIG. 1 (color online). Exchange enhancement factor for the functional in this work (black, solid) compared to PBE functional (dashed, blue). The LDA enhancement factor is identically 1 . 

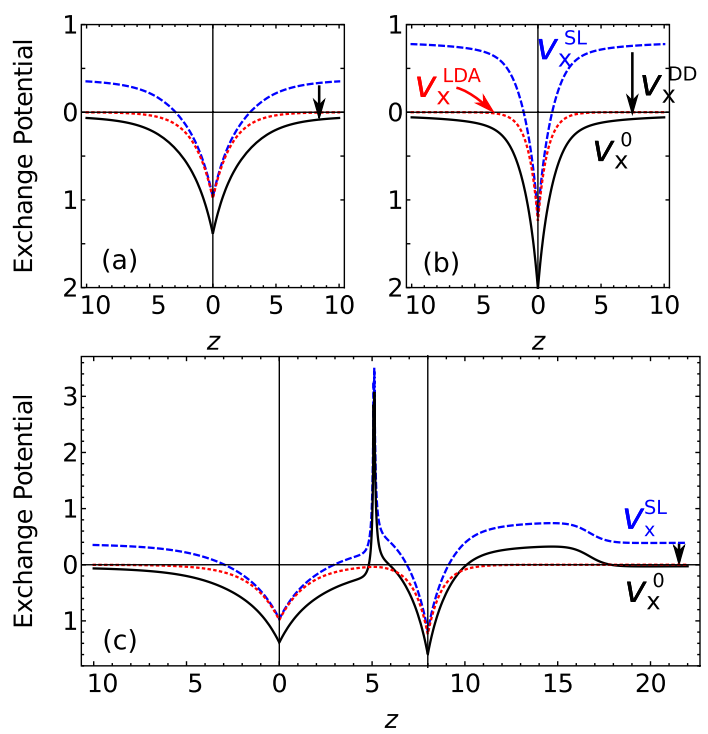

FIG. 2 (color online). Exchange potentials from 1D model densities. (a),(b) Exchange potentials from densities given by individual delta potentials of strength 1 and 2 hartree. (c) Exchange potentials from a density given by both the delta potentials in the same system at, respectively, $z=0$ and 8 bohr. The semilocal potential of this work $v_{x}^{\text {SL }}$ (blue, dashed) has a natural absolute level built in regardless of whether the delta potentials are handled separately, or as parts of a shared system. The constant shift provided by $v_{x}^{\text {DD }}$ (black arrows) brings the potential $v_{x}^{0}$ to zero for all cases but, since it is a constant shift, the relative offset between the parts is retained in the shared system. The LDA potential (red, dotted) provides no such offset.

values. This mechanism is in perfect analogy to the discontinuities of the optimized effective potential [5], yet is here reached with a semilocal functional.

The DD is closely related to a characteristic 'step structure' in the potential (e.g., a step in potential offset between well separated subsystems with different chemical potentials) [3,5]. In Fig. 2 we demonstrate that our potential has this feature by plotting $v_{x}^{\mathrm{SL}}$ and $v_{x}^{0}$ for a test density obtained by filling (as noninteracting) the two bound states in a system with two highly separated delta potentials of asymmetric strength. This model represents two separated 'clusters' with different chemical potentials, i.e., atoms, molecules, or any other clustering of atoms. One cluster is 'stronger' (i.e., a deeper delta potential giving a more strongly bound state) and one is 'weaker.' This model demonstrates many exchange features missing from typical semilocal functionals. [Note: $v_{x}^{\mathrm{DD}}$ is here 3 times Eq. (7), which is the limiting value of the potential for a $1 \mathrm{D}$ system.]

In close vicinity to the center of the delta potentials, $v_{x}^{\mathrm{SL}}$ for the double delta density and for the isolated delta densities are the same, which is necessary for a $v_{x}^{\mathrm{SL}}$ completely semilocal in the density. Since the asymptotic potential for each cluster depends on the highest occupied

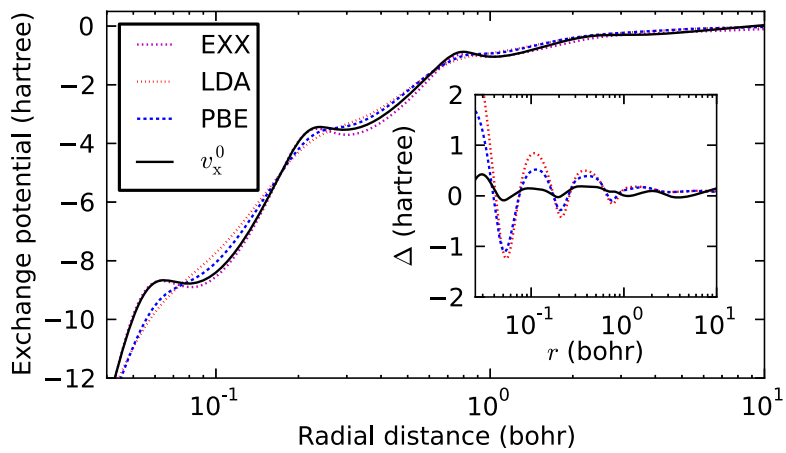

FIG. 3 (color online). Spin-up exchange potential $v_{x}^{0}$ for the $\mathrm{Nb}$ atom as given by different exchange functionals. The inset shows the difference between the functionals and the exact exchange (EXX) potential. Note: the $x$ scale is logarithmic and chosen to show clearly the shell structure. The present functional, like other GGAs, diverges at the atomic nucleus due to the dependence on the Laplacian imposed by the GGA form.

eigenvalue, there is a built-in offset between the two clusters even in isolation, which is retained when the two clusters are placed well separated in the same system. The potential for the stronger cluster is higher due to the $\sqrt{-\epsilon_{I}^{0}}$ dependence. The offset counteracts charge redistribution between the clusters, which should not be present at large separation.

If the clusters are moved closer, the picture of two isolated parts with individual eigenvalue spectra becomes increasingly inaccurate. In the transition into one system and a nonseparable set of eigenvalues, the relative offset between the potentials becomes gradually smaller, and there is a transfer of charge from the weaker to the stronger cluster. However, part of the offset remains. This offset keeps the orbital state that originated on the weak cluster more localized compared to the corresponding computation using the LDA potential, which lacks any such offset.

A single global asymptotic limit is recovered on both sides far away from the delta potentials, and there is a potential peak between the separated clusters. Both these

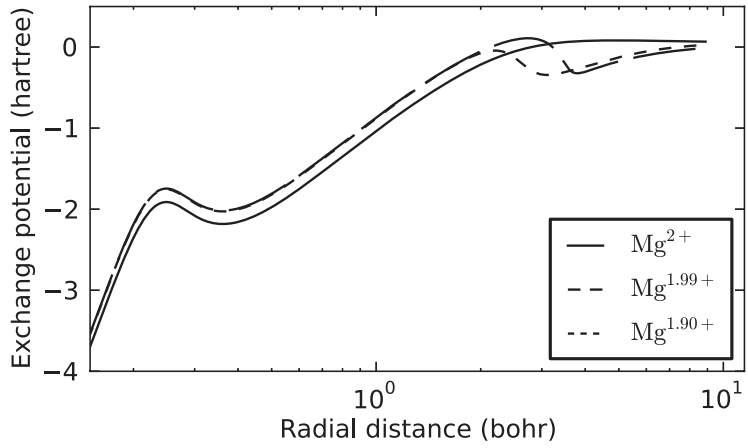

FIG. 4. Spin-up exchange potential $v_{x}^{0}$ for a $\mathrm{Mg}$ ion. When a small fraction of an electron is added to the $\mathrm{Mg}^{+1}$ orbital, the potential shifts discontinuously. 
TABLE I. Energies in hartree related to the ionization of atoms. (Note: the atomic code used only allows this investigation for systems with spherical symmetry.)

\begin{tabular}{lccccc}
\hline \hline & LDAx & EXX & $\left(\epsilon_{I}^{\mathrm{SL}}\right)$ & $\left(\epsilon_{I}^{\mathrm{SL}}+v_{x}^{\mathrm{DD}}\right)$ & $\begin{array}{c}\text { Experimental } \\
\text { values }\end{array}$ \\
\hline $\mathrm{Mg},-\mathrm{HOMO}$ & 0.142 & 0.253 & 0.141 & 0.237 & 0.281 \\
$\mathrm{Mg}, I_{\Delta \mathrm{SCF}}$ & 0.238 & 0.242 & & 0.215 & 0.281 \\
$\mathrm{Ca},-\mathrm{HOMO}$ & 0.112 & 0.195 & 0.109 & 0.196 & 0.225 \\
$\mathrm{Ca}, I_{\Delta \mathrm{SCF}}$ & 0.188 & 0.187 & & 0.160 & 0.225 \\
$\mathrm{Kr},-\mathrm{HOMO}$ & 0.300 & 0.524 & 0.284 & 0.410 & 0.514 \\
$\mathrm{Cd},-\mathrm{HOMO}$ & 0.167 & 0.265 & 0.139 & 0.234 & 0.331 \\
\hline \hline
\end{tabular}

features correspond closely to the exact potential $[4,20,39,40]$. We emphasize that all these crucial potential features are reproduced in this work with a normal semilocal GGA-type expression using only the density and density gradient. The only purpose of $v_{x}^{\mathrm{DD}}$ is a final adjustment of the zero of the potential such that the global asymptotic limit $v_{x}^{0} \rightarrow 0$.

We have implemented $E_{x}^{\mathrm{SL}}$ and $v_{x}^{\mathrm{SL}}$ in a code for atoms originating from Ref. [41]. This code solves the KS equations on a logarithmic radial grid, which makes it possible to reach a converged all-electron result without basis set issues. We find that the functional strongly improves the shell structure of atoms compared to usual semilocal functionals, as shown for $\mathrm{Nb}$ in Fig. 3. The improved structure is built into $v_{x}^{\mathrm{SL}}$, and the uniform shift $v_{x}^{\mathrm{DD}}$ aligns the asymptote to make $v_{x}^{0} \rightarrow 0$.

We make a closer examination of the DD in an atomic system in Fig. 4. As we take the charge of a $\mathrm{Mg}$ ion from +2 to $+(2-\delta)$, the zero-aligned potential $v_{x}^{0}$ makes a discontinuous uniform shift. The shift is roughly $1 / 3$ of that found for EXX (not shown). Note that we do not need to strive to reproduce the full DD of EXX, since semilocal DFT relies on effectively including some static correlation in the exchange functional $[5,42]$ and the total DD is known to be smaller than the one of EXX [43].

A further hallmark deficiency of usual semilocal functionals is addressed in Table I. The HOMO eigenvalue (relative to its proper zero, i.e., $\epsilon_{I}^{\mathrm{SL}}+v_{x}^{\mathrm{DD}}$ ) from our approach is much closer to the one of EXX and the experimental ionization potential than found for usual semilocal approaches. Total energies without correlation contributions are of limited accuracy, yet it is noteworthy that the agreement between the HOMO eigenvalue and the difference between total energies of the neutral and ionized system $I_{\Delta_{\mathrm{SCF}}}$ is much improved.

We have also implemented $E_{x}^{\mathrm{SL}}$ in the computational code Exciting, a full-electron linearized augmented plane wave (LAPW) code $[44,45]$. We have calculated the band structures for $\mathrm{Si}$ and $\mathrm{Cu}$ at experimental lattice constants, shown in Fig. 5, and also for Ge (not shown) [46]. The Si and $\mathrm{Ge}$ band structures reproduce well the general features of EXX [43]. The band gap of functionals with a nonzero

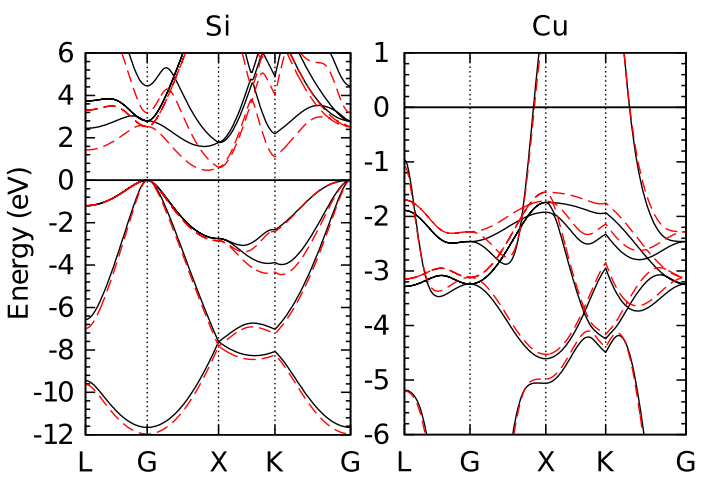

FIG. 5 (color online). Band structure of $\mathrm{Si}$ and $\mathrm{Cu}$ for the functional in this work (black) compared to LDA (dashed red). For $\mathrm{Si}$ the $\mathrm{KS}$ gap is $1.6 \mathrm{eV}$, i.e., much larger than the $\mathrm{KS}$ gap from LDA. The $\mathrm{Cu}$ band structure shows a small but important shift that brings the localized $d$ orbitals down in energy relative to other states (the flatter bands at -1 to $-4 \mathrm{eV}$ are the ones dominated by $d$ states). (Results using PBE at the same, experimental, volume are virtually indistinguishable from LDA.)

contribution from the DD $\Delta_{\mathrm{xc}}$ needs careful consideration $[5,43,47]$, and there is no reason to expect the KS gap $\Delta_{\mathrm{KS}}$ from $v_{x}^{\mathrm{SL}}$ to accurately reproduce experimental $0 \mathrm{~K}$ band gaps (for $\mathrm{Si}, \approx 1.17 \mathrm{eV}$ and for $\mathrm{Ge}, \approx 0.74 \mathrm{eV}$ ), given both the unclear contribution from $\Delta_{\mathrm{xc}}$ and that our focus has been on reproducing features previously not seen in this type of functional, rather than a complete exchange-correlation functional optimized for accuracy. Nevertheless, normal semilocal functionals have $\Delta_{\mathrm{xc}}=0$, and the total value $\Delta=\Delta_{\mathrm{KS}}+\Delta_{\mathrm{xc}}$ tends to be much too small. The present functional addresses this issue in a similar way as EXX, with much increased KS gaps. We find $\Delta_{\mathrm{KS}}^{\mathrm{Si}} \approx 1.6 \mathrm{eV}$ and $\Delta_{\mathrm{KS}}^{\mathrm{Ge}} \approx 0.7 \mathrm{eV}$. It is especially encouraging to find the functional to fully rectify the qualitative deficiency of a vanishingly small gap for $\mathrm{Ge}$ seen with many semilocal functionals.

Figure 5 also shows the band structure of $\mathrm{Cu}$, a characteristic example of incorrectly delocalized orbitals in semilocal DFT. Localized orbitals, i.e., the $d$ bands, move down in energy and other bands change in accordance to GW results, but with less magnitude [48]. Hence, the present functional allows for a qualitative correction to band structure that has previously been unavailable within KS DFT at the current level of computational expense.

In conclusion, this Letter has presented a closed-form GGA-type semilocal functional that addresses over delocalization, charge transfer, and the description of band structure in semilocal DFT by reproducing exchange features previously unavailable within KS DFT without using orbital functionals.

R. A. acknowledges support from the Swedish Research Council (VR), Grant No. 621-2011-4249 and the Linnaeus Environment at Linköping on Nanoscale Functional Materials (LiLi-NFM) funded by VR. S. K. acknowledges support by the German-Israeli Foundation. 
[1] P. Hohenberg and W. Kohn, Phys. Rev. 136, B864 (1964).

[2] W. Kohn and L. J. Sham, Phys. Rev. 140, A1133 (1965).

[3] J. P. Perdew, R. G. Parr, M. Levy, and J.L. Balduz, Phys. Rev. Lett. 49, 1691 (1982).

[4] O. V. Gritsenko and E. J. Baerends, Phys. Rev. A 54, 1957 (1996).

[5] S. Kümmel and L. Kronik, Rev. Mod. Phys. 80, 3 (2008).

[6] J. P. Perdew and Y. Wang, Phys. Rev. B 33, 8800 (1986).

[7] R. van Leeuwen and E. J. Baerends, Phys. Rev. A 49, 2421 (1994).

[8] A. Lembarki, F. Rogemond, and H. Chermette, Phys. Rev. A 52, 3704 (1995).

[9] D. J. Tozer and N. C. Handy, J. Chem. Phys. 109, 10180 (1998).

[10] O. V. Gritsenko, P.R. T. Schipper, and E. J. Baerends, Chem. Phys. Lett. 302, 199 (1999).

[11] D. J. Tozer, J. Chem. Phys. 112, 3507 (2000).

[12] M. Grüning, O. V. Gritsenko, S. J. A. van Gisbergen, and E. J. Baerends, J. Chem. Phys. 114, 652 (2001).

[13] A. P. Gaiduk and V. N. Staroverov, J. Chem. Phys. 128, 204101 (2008).

[14] A. D. Becke and E. R. Johnson, J. Chem. Phys. 124, 221101 (2006).

[15] R. Armiento, S. Kümmel, and T. Körzdörfer, Phys. Rev. B 77, 165106 (2008).

[16] F. Tran, P. Blaha, and K. Schwarz, J. Phys. Condens. Matter 19, 196208 (2007).

[17] F. Tran and P. Blaha, Phys. Rev. Lett. 102, 226401 (2009).

[18] S. Pittalis, E. Räsänen, and C. R. Proetto, Phys. Rev. B 81, 115108 (2010).

[19] M. J. T. Oliveira, E. Räsänen, S. Pittalis, and M. A. L. Marques, J. Chem. Theory Comput. 6, 3664 (2010).

[20] A. Karolewski, R. Armiento, and S. Kümmel, J. Chem. Theory Comput. 5, 712 (2009).

[21] See Supplemental Material at http://link.aps.org/ supplemental/10.1103/PhysRevLett.111.036402 for more details on the derivation of the functional and the results of volume relaxation of bulk Al.

[22] T. Kreibich, S. Kurth, T. Grabo, and E. K. U. Gross, Adv. Quantum Chem. 33, 31 (1998).

[23] http://www.wolfram.com/mathematica/.

[24] L. Pollack and J. P. Perdew, J. Phys. Condens. Matter 12, 1239 (2000).

[25] Z. Qian, Phys. Rev. B 85, 115124 (2012).

[26] P. R. Antoniewicz and L. Kleinman, Phys. Rev. B 31, 6779 (1985).
[27] L. Kleinman and S. Lee, Phys. Rev. B 37, 4634 (1988).

[28] A. D. Becke, J. Chem. Phys. 98, 1372 (1993).

[29] J. P. Perdew, M. Ernzerhof, and K. Burke, J. Chem. Phys. 105, 9982 (1996).

[30] M. Levy, N. H. March, and N. C. Handy, J. Chem. Phys. 104, 1989 (1996).

[31] A. Seidl, A. Görling, P. Vogl, J. A. Majewski, and M. Levy, Phys. Rev. B 53, 3764 (1996).

[32] R. Neumann, R. H. Nobes, and N. C. Handy, Mol. Phys. 87, 1 (1996).

[33] V. I. Anisimov, J. Zaanen, and O. K. Andersen, Phys. Rev. B 44, 943 (1991).

[34] V.I. Anisimov, I. V. Solovyev, M. A. Korotin, M. T. Czyżyk, and G. A. Sawatzky, Phys. Rev. B 48, 16929 (1993).

[35] A. I. Liechtenstein, V. I. Anisimov, and J. Zaanen, Phys. Rev. B 52, R5467 (1995).

[36] S. L. Dudarev, G. A. Botton, S. Y. Savrasov, C. J. Humphreys, and A.P. Sutton, Phys. Rev. B 57, 1505 (1998).

[37] M. Levy and J. P. Perdew, Phys. Rev. A 32, 2010 (1985).

[38] J. P. Perdew, K. Burke, and M. Ernzerhof, Phys. Rev. Lett. 77, 3865 (1996).

[39] A. Makmal, S. Kümmel, and L. Kronik, Phys. Rev. A 83, 062512 (2011).

[40] D. G. Tempel, T. J. Martínez, and N. T. Maitra, J. Chem. Theory Comput. 5, 770 (2009).

[41] J. D. Talman and W.F. Shadwick, Phys. Rev. A 14, 36 (1976).

[42] R. Armiento and A. E. Mattsson, Phys. Rev. B 68, 245120 (2003).

[43] M. Städele, M. Moukara, J. A. Majewski, P. Vogl, and A. Görling, Phys. Rev. B 59, 10031 (1999).

[44] D. J. Singh, Planewaves, Pseudopotentials, and the LAPW Method (Kluwer Academic Publishers, Boston, 1994).

[45] http://exciting-code.org/.

[46] Computational parameters for the LAPW scheme are as follows: all calculations used $15 \times 15 \times 15 k$ points, 60 empty bands, and muffin-tin radii for all species of $R=2.0 \mathrm{bohr}$ and as cutoff for plane waves in the interstitial regions $k_{\max }=9.0 / R$. The band structures are aligned on the Fermi energy, taken as the HOMO state.

[47] L. Kronik, T. Stein, S. Refaely-Abramson, and R. Baer, J. Chem. Theory Comput. 8, 1515 (2012).

[48] A. Marini, G. Onida, and R. Del Sole, Phys. Rev. Lett. 88, 016403 (2001). 